\title{
Factor Técnico de la Finalización De Éxito - Fútsal
}

\section{Technical Factor of Successful Finishing - Fútsal}

\author{
Darío Augusto Cuasapud-Arroyave' ${ }^{1}$; ; Brenda Viviana Sánchez-Mora' ${ }^{1}$ (D) \\ 'Universidad del Valle. Cali - Valle del Cauca, Colombia; dario.cuasapud@correounivalle.edu.co; \\ brevisamo@gmail.com
}

Cómo citar: Cuasapud-Arroyave, D.A.; Sánchez-Mora, B.V. 2021. Factor Técnico de la Finalización De Éxito - Fútsal. Rev. Digit. Act. Fis. Deport. 7(1):e1522. http://doi.org/10.31910/rdafd.v7.n1.2021.1522

Artículo de acceso abierto publicado por Revista Digital: Actividad Física y Deporte, bajo una licencia Creative Commons CC BY-NC 4.0

Publicación oficial de la Universidad de Ciencias Aplicadas y Ambientales U.D.C.A, Institución de Educación Superior Acreditada de Alta Calidad por el Ministerio de Educación Nacional.

Recibido: Abril 17 de 2020 Aceptado: Noviembre 15 de 2020 Editado por: Álvaro José Gracia Díaz

\section{RESUMEN}

Introducción: Los estudios mixtos que descubren los eventos del proceso y, a la vez, otorgan explicaciones, se han apoyado recurrentemente en análisis notacionales (observacionales), determinando nuevos indicadores de rendimiento. En el presente estudio, se escoge el aspecto fundamental de la técnica. Objetivo general: Analizar el factor técnico individual ofensivo con balón del ejecutor de la Finalización de Éxito (F.É.), más conocido como Gol, estimando el desempeño técnico del jugador ejecutor, por medio de análisis de las relaciones asociativas intrafactor. Metodología: Se basó en los métodos observacionales, a través de videos oficiales de FIFA; 352 F.É., 52 partidos y 24 selecciones nacionales, observando las variables del Factor Técnico: 1) Habilidades técnicas, 2) Tipos de Tiro, 3) Superficies de Contacto, 4) Dominancia y 5) Perfil. Se registró, en instrumentos de observación, adaptados de tesis doctorales y el software de video análisis Longo Match 1.0., recodificando y analizando en programa SPSS 20, en estadística descriptivas y pruebas Chi-cuadrado de Pearson (x2) y coeficientes de comprobación V-Cramer. Resultados: Se encontraron y se cuantificaron las frecuencias y los porcentajes del factor técnico, considerando relaciones asociativas significativas e infiriendo las distribuciones de alcance y de patrones, confrontándolos con expertos del rendimiento deportivo, en análisis técnico de la modalidad del fútsal. Conclusiones: Se determinaron los parámetros del dominio de las habilidades técnicas requeridas por el deportista ejecutor, en la consecución de la F.É., determinando la estimación de desempeño técnico individual ofensivo con balón en la F.É., otorgando referencias a entrenadores e investigadores, del componente técnico-táctico del jugador de fútsal.

Palabras clave: Técnica; Gol; Fútsal; Observacional; Rendimiento deportivo.

\section{ABSTRACT}

Introduction: Mixed studies that discover the events of the process and at the same time provide explanations, have recurrently relied on notational (observational) analyzes; determining new performance indicators. In the present study, the fundamental aspect of the technique is chosen. For this reason. Course Objective: To analyze the individual offensive technical factor with the ball of the executor of the Success End (F.É.) better known as Gol; estimating the technical performance of the 
executing player through analysis of the associative relationships within the factor. Methodology: used was based on observational methods, through official FIFA videos; 352 F.É., 52 games and 24 national teams; observing the variables of the Technical Factor: Technical skills, Types of Shot, Contact Surfaces, Dominance and Profile. It was registered in observation instruments adapted from doctoral theses and Longo Match 1.0 video analysis software. recoding and analyzing in the SPSS 20 program, in descriptive statistics and Pearson's Chi-square tests (x2) and V-Cramer verification coefficients. Results: Found quantified the frequencies and percentages of the technical factor, considering significant associative relationships and inferring the distributions of range and patterns. Confronting them with sports performance experts in technical analysis of the Futsal modality. Conclusions: Determining parameters of mastery of the technical skills required by the executing athlete, in achieving the F.É. Determining the estimation of individual offensive technical performance with the ball in the F.É., giving references to coaches and researchers, of the technical-tactical component of the Futsal player.

Keywords: Technique; Goal; Futsal; Observational; Sports performance.

\section{INTRODUCCIÓN}

En la Teoría del Entrenamiento Deportivo, cada actividad deportiva es considerada importante de ser estudiada, como lo son sus respectivas modalidades. La disciplina deportiva fútbol posee, entre sus modalidades, el Fútbol Sala FIFA o bien conocido como Fútsal FIFA. En los estudios investigativos más conocidos dentro del fútsal, se encuentran los análisis de los entrenadores, análisis de aspectos psicológicos de jugadores, análisis técnicos-tácticos de equipos, entre otros (Garganta, 2009; Moore et al. 2014).

Uno de los campos de estudio y desarrollo del análisis del rendimiento en deportes colectivos de colaboración-oposición y de invasión de campo contrario (Gonçalves et al. 2014) son las investigaciones que han seguido ahondando en brechas investigativas con relación a temas de efectividad de factores técnico-tácticos (Agras et al. 2016), los cuales, tienen como común denominador un direccionamiento con relación al éxito de las finalizaciones en las secuencias ofensivas o más conocidas, como gol (Puente et al. 2004; Santana et al. 2013; Sarmento et al. 2016).

Los estudios que se establecen desde observaciones de secuencias finales para el disparo permiten visualizar a las F.É., como un elemento más de todo, pero, a su vez, es la parte más importante del contexto del juego (Abdel-Hakim, 2014). Esto hace que se planteen interrogantes sobre los factores que influyen en la F.É., desde distintos ángulos (técnicos, tácticos, físicos, psicológicos, entre otros) y, para ello, los análisis de equipos, jugadores y acciones de juego, en contexto de competencia, son el escenario más apropiado (Cuasapud \& Hurtado, 2018).

Apoyados en la metodología observacional de Blanco et al. (2001), se realiza el estudio de videos resumen de la página oficial de FIFA, donde se visualizan las 352 F.É., en 52 partidos realizados por los 24 equipos representativos de las naciones clasificadas. El estudio, se desarrolla en el factor de análisis técnico: Habilidad Técnica, Tipo de Disparo, Superficie de Contacto, Dominancia y Perfil.

La captura de los registros y análisis de información, se hace bajo el Software de Video Análisis Longo Match 1.0. y la recodificación, en el programa estadístico SPSS 20, donde se obtienen análisis de frecuencias, permitiendo descubrir relaciones, secuencias y patrones recurrentes. El estudio concluye con la estimación del desempeño técnico requerido del jugador ejecutor de las F.É. de la VIII Copa Mundial de Fútsal FIFA, Colombia 2016, por medio de la identificación del factor técnicos individual ofensivo, destacando las distribuciones y las relaciones asociativas (De Bortoli et al. 2001).

\section{MATERIALES Y MÉTODOS}

Población y muestra. Para este caso, se decidió registrar una unidad experimental completa, es decir, se tomaron los 352 F.É., ocurridas en los 52 juegos realizados por las 24 naciones, que asistieron a la VIII Copa Colombia 201,6 entre el 10 de septiembre al 1 de octubre de 2016, en Colombia. Se registraron la totalidad de las acciones en el factor técnico con sus respectivas variables. En total, se obtuvieron 1.760 datos registrados y analizados en 5 variables técnicas (Tabla 1). 
Tabla 1. Características de las Finalizaciones de Éxito por fase del torneo.

\begin{tabular}{|l|c|c|c|}
\hline F.É/FASE Equipos & Partidos & F.E. & Eventos \\
\hline Grupos 24 & 36 & 245 & 1.225 \\
\hline Octavos 16 & 8 & 55 & 275 \\
\hline Cuartos 8 & 4 & 25 & 125 \\
\hline Semifinal-Final 4 & 4 & 27 & 135 \\
\hline TOTAL 24 & $\mathbf{5 2}$ & $\mathbf{3 5 2}$ & $\mathbf{1 . 7 6 0}$ \\
\hline
\end{tabular}

Tipo de estudio y procedimiento. El método utilizado es cuantitativo-descriptivo-trasversal, el cual, estudia el número de veces que se repite el comportamiento de los goles o F.É. en cada partido y por equipo. Se apoya en la metodología observacional de Lapresa et al. (2015), Liu et al. (2016), Leite (2013), que se basa en el seguimiento nomotético (de equipo a equipo), inter-sesional (partido a partido), intra-sesional (F.É. a F.É), en el evento mundialista. La investigación pasó por las siguientes fases: 1) Variables de análisis y sus categorías, 2) Recolección de la muestra, 3) Evaluación de la fiabilidad de la información, 4) Codificación de la información, 5) Tratamiento, 6) Análisis de los datos en el procedimiento estadístico, 7) Desarrollo de los resultados 8) Discusión y conclusiones del estudio.

Definición de variables. Para la definición de variables que se utilizan en el estudio, se optó por ubicarlas en el factor técnico, con sus respectivas variables. Cada variable posee un punto estratégico en la resolución de los objetivos y están categorizadas de forma numérica, para facilitar su registro y tratamiento estadístico. El factor técnico utiliza variables independientes que describen, de manera directa, la habilidad, la superficie, el tipo de tiro o disparo, la dominancia y el perfil que utiliza cada ejecutor de la F.É. requerida (Tabla 2).

Tabla 2. Factor Técnico de la Finalización de Éxito.

\begin{tabular}{|c|c|c|c|}
\hline \multicolumn{2}{|c|}{ Habilidad técnica } & \multicolumn{2}{|c|}{$\begin{array}{c}\text { Superficies de } \\
\text { contacto }\end{array}$} \\
\hline Denominación & Sigla & Denominación & Sigla \\
\hline Conducción & (CND) & Cabeza & $(\mathrm{CAB})$ \\
\hline Control & (CON) & Combinación & $(\mathrm{CCO})$ \\
\hline Finta & $(\mathrm{FIN})$ & Empeine & (CEM) \\
\hline $\begin{array}{l}\text { Manejo del } \\
\text { balón }\end{array}$ & (MAN) & Borde externo & (CEX) \\
\hline $\begin{array}{l}\text { Otros } \\
\text { contactos }\end{array}$ & (OTH) & Borde interno & $(\mathrm{CIN})$ \\
\hline Recuperación & (REC) & Otras & (COT) \\
\hline Regate & (REG) & Planta & (CPL) \\
\hline Disparo & (DP) & Punta & (CPU) \\
\hline \multicolumn{2}{|l|}{ Tipo de disparo } & \multicolumn{2}{|c|}{ Dominación } \\
\hline Denominación & Sigla & Denominación & Sigla \\
\hline Remate & (REM) & Derecha & (DER) \\
\hline Toque & (TOQ) & Izquierda & $(I Z Q)$ \\
\hline Otro & (OTR) & Otra & (OTR) \\
\hline Autogol & (AUT) & & \\
\hline \multicolumn{2}{|c|}{ Perfil } & & \\
\hline Denominación & Sigla & & \\
\hline Cambiado & (PCAM) & & \\
\hline Propio & (PPRO) & & \\
\hline Frontal & (PFRO) & & \\
\hline
\end{tabular}

Nota: Adaptado de Análisis técnico-táctico de la fase ofensiva de la selección española de fútbol sala (Eurocopa, 2010)”, por Álvarez Kurogi, 2015. Tesis doctoral sin publicar.

Universidad de La Rioja. Logroño. 
- Habilidades: Las conductas que desarrolla el jugador para resolver de forma racional y económica un problema gestual determinado (De Bortoli et al. 2001).

- $\quad$ Superficies de contacto: Son las partes anatómicas que, con mayor frecuencia y de manera normal, se utilizan para el manejo del balón o móvil (Álvarez, 2015).

- $\quad$ Tipos de disparos: Tipo de técnica utilizada para la F.É., se puede realizar de manera directa (a través de un remate), indirecto (tocando el balón), por otra circunstancia (rebote u otro) o simplemente que lo realice un mismo defensor, para este caso, se denomina autogol (Marchi et al. 2010).

- Dominancia: Determina la extremidad utilizada en la acción técnica final, para el disparo que termina en gol o F.É. Puede ser pierna izquierda, derecha y, en caso diferente, se cataloga como otro (De Bortoli et al. 2002; Álvarez, 2015).

- $\quad$ Perfil: Alude al perfil que utiliza en ejecutor al realizar el disparo a portería que termina en gol. Puede ser propio, si la ejecución es con pierna dominante (ejemplo: derecho) y por el corredor derecho; se considera perfil cambiado, cuando dispara con la pierna dominante (derecha), pero por el corredor izquierdo, es decir, contrario. Se considera frontal, cuando dispara por el corredor central, indiferente de la dominancia (derecha o izquierda) (De Bortoli et al. 2002).

Materiales. Para la recolección de información, el proceso de registro y almacenamiento de la misma, se tuvo en cuenta los siguientes materiales de recolección. Como videos de la página oficial FIFA (2016), ordenador portátil Lenovo, disco duro externo de $1 \mathrm{~Tb}$, información oficial de Estadística por juego, software de apoyo Longo Match 1.0., tabla de recolección en Excel y programa SPSS 20.

Instrumentos de análisis. Se lleva a cabo, utilizando el programa SPSS 20 (Licencia Institución Universitaria Escuela Nacional del Deporte), en el cual, se exhiben los resultados de eventos observados, organizándolos por medio de tablas e ilustraciones de cada uno de los valores de interés estadístico. Tratándose de variables categóricas, se comprueban los resultados por medio de la prueba de Chi-cuadrado de Pearson $\left(x^{\wedge} 2\right)$, sujetando cada variable principal e independiente de los dos factores en un análisis pareado (por pares) y éstas, a su vez, con las variables restantes. La $V$ de Cramer es una corrección, que se puede aplicar al coeficiente Chi Cuadrado, lo cual, permite obtener un índice con valor máximo (que indica la mayor asociación entre variables) igual a 1 (el valor mínimo es 0 , que indica NO asociación) (Nevill et al. 2002).

\section{RESULTADOS}

Análisis de frecuencia del factor técnico para la F.É. Las F.É. que se lograron en la VIII Copa Mundial de Futsal 2016, se realizaron con diferentes habilidades técnicas. Fundamentalmente, se entiende que el ejecutor realiza la F.É. con una sola habilidad $(59,65 \%)$. Al detallarse la frecuencia, se presenta que el aspecto técnico requerido por el ejecutor de la F.É. utilizó en alto porcentaje la habilidad de disparo, el tipo de disparo remate, la superficie de contacto empeine, la dominancia derecha y el perfil contrario (Tabla 3).

Tabla 3. Multitabla de frecuencia del factor técnico de las Finalizaciones de Éxito.

\begin{tabular}{|c|c|c|c|}
\hline \multicolumn{2}{|c|}{$\begin{array}{c}\text { Superficie de contacto con la que se } \\
\text { realizó la F.É. }\end{array}$} & \multicolumn{2}{|c|}{ Perfil con el que se realizó la F.É. } \\
\hline $\begin{array}{l}\text { Superficie de } \\
\text { contacto }\end{array}$ & Porcentaje & Perfil & Porcentaje \\
\hline Cabeza & $1,42 \%$ & Cambiado & $44,60 \%$ \\
\hline Empeine & $51,42 \%$ & Propio & $21,59 \%$ \\
\hline Borde externo & $1,42 \%$ & Frontal & $33,81 \%$ \\
\hline Borde interno & $31,82 \%$ & \multicolumn{2}{|c|}{ Dominancia en la realización de la F.É. } \\
\hline Otras & $4,26 \%$ & Dominancia & Porcentaje \\
\hline Planta & $0,28 \%$ & Derecha & $55,68 \%$ \\
\hline Punta & $9,09 \%$ & Izquierda & $39,77 \%$ \\
\hline Combinación & $0,28 \%$ & Otra & $4,55 \%$ \\
\hline \multicolumn{2}{|c|}{$\begin{array}{c}\text { Tipo de disparo con que se realizó la } \\
\text { F.É. }\end{array}$} & \multicolumn{2}{|c|}{ Habilidad 1 con la que se realizó la F.É. } \\
\hline Tipo de disparo & Porcentaje & Habilidad 1 & Porcentaje \\
\hline Remate & $76,14 \%$ & Disparo & $96,31 \%$ \\
\hline Toque & $20,17 \%$ & Otra & $1,14 \%$ \\
\hline Otro & $1,14 \%$ & No (Autogol) & $2,56 \%$ \\
\hline Autogol & $2,56 \%$ & & \\
\hline
\end{tabular}


Análisis de relación asociativa entre variables Intra - Factor Técnico. En la figura 1, se muestra la relación asociativa entre variables del factor técnico para las F.É. Se presenta cómo se destacó en cada variable en análisis particular. Las características técnicas de las F.É. que fueron requeridos por su ejecutor, en la Copa Mundial 2016 de Fútsal. Se identificaron por la habilidad de disparo $(96,31 \%)$, por un tipo de disparo remate $(76,14 \%)$, el empeine como la superficie de contacto (51,42\%), con dominancia derecha $(55,68)$ y perfil cambiado utilizado $(44,60 \%)$.

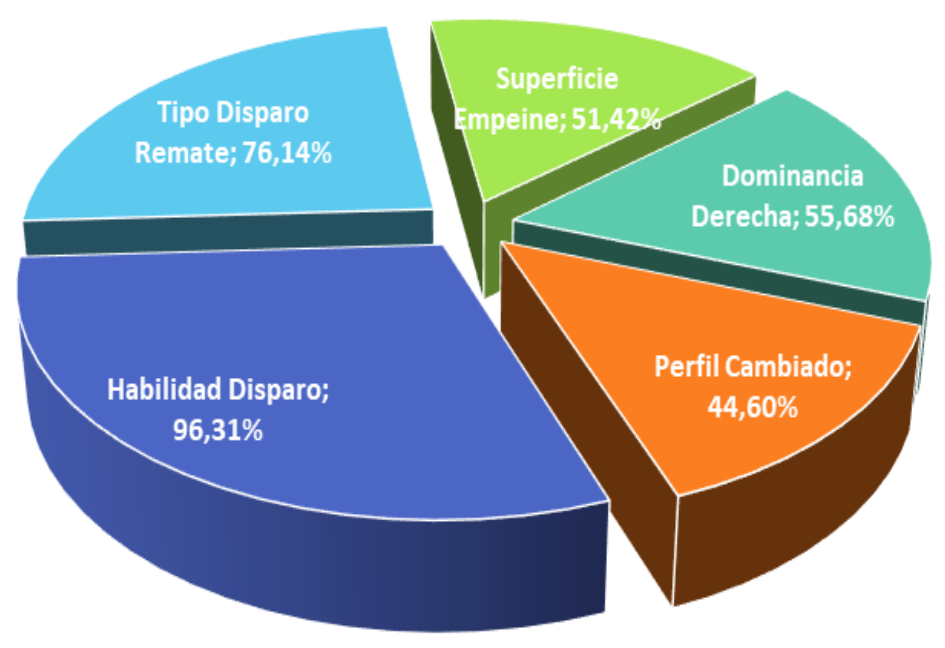

Figura 1. Relación asociativa para las F.É.

En la figura 2, se muestra la relación asociativa entre variables del factor técnico para las F.É. con una habilidad, presenta cómo se destacó cada variable en su análisis particular. Cuando se realiza la F.É. sin habilidad previa, el disparo ocupa un primer renglón $(59,65 \%)$ de las situaciones ofensivas. Entretanto que, el tipo de disparo que se ha denominado "remate" $(73,81 \%)$, la aparición de la superficie de contacto borde interno $(44,76 \%)$ al lado de la superficie de contacto empeine (44,29\%), para el disparo. Se afirma la dominancia derecha, aumentando su aparición $(57,14 \%)$ y en el perfil ocurre algo similar $(45,24 \%)$.

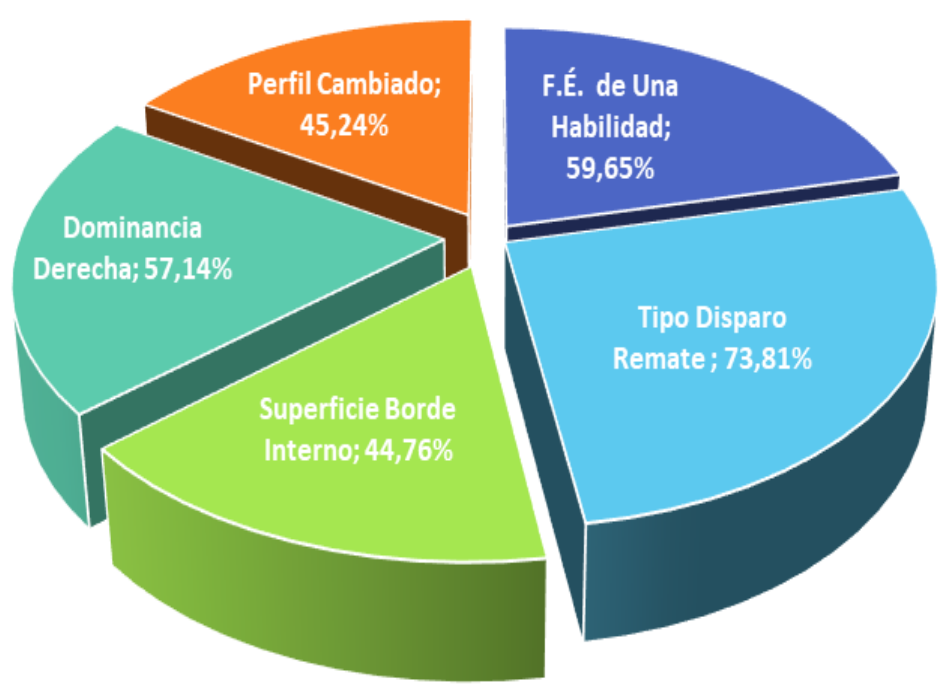

Figura.2. Relación asociativa de F.É con H1. 
Análisis de correlación según efecto asociativo entre variables Intra - Factor Técnico. En este sentido, se propone realizar la prueba de independencia Chi-cuadrado, la cual, consiste en comprobar si dos variables cualitativas están relacionadas entre sí. Adicional para las variables que resulten relacionadas, se obtiene una medida, para identificar la intensidad de la relación, que es V-Cramer, que tiene la siguiente escala:

- $\quad$ 0-0.1.00 No hay relación efecto entre las variables

- $\quad 0.10-0.30$ Efecto pequeño entre las variables

- $\quad 0.30-0.50$ Efecto moderado entre las variables

- 0.50-1.00 Efecto grande entre las variables
A continuación, se presentan los resultados para las variables que mostraron relación, clasificados según la intensidad de la relación, expresada según V-Cramer. De los nueve pares de variables (Tabla 4), que mostraron mayor efecto encontramos como la habilidad técnica disparo (DP), se relacionaba directamente con el tipo de disparo remate (REM), la superficie de contacto uno (CEM) mostraron la más alta relación $(0,778)$, comprobando los resultados del análisis del factor técnico.

Tabla 4. Prueba Chi-cuadrado de independencia - V-Cramer, con tipo de Efecto.

\begin{tabular}{|l|c|c|c|c|}
\hline \multicolumn{1}{|c|}{ Variables } & $\begin{array}{c}\text { Chi- } \\
\text { Cuadrado }\end{array}$ & $\begin{array}{c}\text { Valor- } \\
\mathbf{p}\end{array}$ & V-Cramer & Efecto \\
\hline Habilidad - Tipo de Disparo & 704 & 0 & 1 & Grande \\
\hline Habilidad - Superficie & 213,253 & 0 & 0,778 & Grande \\
\hline Superficie - Dominancia & 295,562 & 0 & 0,648 & Grande \\
\hline Dominancia - Tipo de Disparo & 196,757 & 0 & 0,529 & Grande \\
\hline Tipo de Disparo - Superficie & 291,202 & 0 & 0,525 & Grande \\
\hline Habilidad - Dominancia & 194,119 & 0 & 0,525 & Grande \\
\hline Perfil - Superficie & 55,48 & 0 & 0,281 & Pequeño \\
\hline Perfil - Dominancia & 40,538 & 0 & 0,24 & Pequeño \\
\hline Perfil - Tipo de Disparo & 28,852 & 0 & 202 & Pequeño \\
\hline
\end{tabular}

El análisis del factor técnico requerido por el ejecutor de la F.É. reveló un patrón usual: La formación de patrones de comportamientos en las jugadas de atacantes-defensor en juegos de equipos (Headrick et al. 2012) son aceptados por la comunidad científica del deporte, por ello, el estudio arroja lo siguiente.

- $\quad$ En las F.É. en general: utiliza una sola Habilidad (59,65\%), Disparo (D) $(96,31 \%)$, Tipo de Disparo Remate (REM) (76,14\%), Superficie de Contacto Empeine (CEM) (51,42\%), Dominancia Derecha (DDER) (55,68\%), Perfil Cambiado (PCAM) (44,60\%).

- $\quad$ F.É. Una Habilidad: (CINT) $(44,76 \%)$ - (EMP) $(44,29 \%)$,

\section{DISCUSIÓN}

\section{Análisis del factor técnico requerido por el ejecutor en la F.É.}

Habilidad Técnica. En el factor técnico, se reconoce que el fundamento técnico más utilizado en el juego es el pase (Álvarez et al. 2018), luego, el control 28,9\% (Álvarez, 2015) y en tercer renglón, se encuentra la habilidad técnica para la F.É., el disparo $(59,65 \%)$, lo cual, continúa con datos similares para los principales autores del tema (Alves, 2010; Duarte, 2008). A pesar de que el disparo como habilidad técnica, representa solo el 11,7\% (Álvarez, 2015), del total de las habilidades técnicas utilizadas en 
la fase ofensiva del juego, el disparo a portería o tiro, representa la mejor opción para el ejecutante de la F.É. (96,3\%), en concordancia con Puente et al. (2004), en el análisis de 1.771 goles o F.É., en la división de honor de España 2002 (90\%) y al (97,6\%), descrito por Álvarez (2015), en su análisis de la selección de España en la Eurocopa 2010.

Tipo de Disparo. El tipo de disparo o tiro, o más conocido como remate. Para este ejercicio, se creó la clasificación para la habilidad técnica del disparo, denominada tipo de disparo (96,31\%), que incluye el remate (REM) y el toque (TOQ). Marchi et al. (2010) analizó los goles o F.É., en contexto de transiciones ofensivas o contraataques $(63,8 \%)$ y han considerado la misma importancia, al estudio del tipo de disparo en los goles o F.É. Es importante resaltar que la clasificación que se le dio en el presente documento investigativo al tipo de disparo, no tiene antecedentes, es decir, los autores cuando describen el tipo de disparo, solamente lo dividen en directos e indirectos, dejando en el segundo renglón de indirectos a los autogoles y los rebotes, principalmente, mas no realizan una diferencia a la hora de la ejecución, si es un gesto técnico de remate $(76,14 \%)$ o si es un toque por ubicación $(20,17 \%)$.

Para este documento, se ha determinado al disparo directo se entienda solo como el gesto de remate y el indirecto, como el disparo, pero que se ejecuta como un toque, es decir, que el disparo con lo que se hace la F.É., se realizará como un pase con borde interno, en el momento de asegurar la F.É. o, por el contrario, la velocidad del pase previo a la F.É. es tan fuerte o se realiza a tan alta velocidad, que el ejecutante debe realizar más que gesto técnico de remate; es un ajuste postural y mecánico de la acción técnica, que lo reduce a optimizar dicho gesto, en solo una colocación de la superficie de contacto, más que en la ejecución del gesto técnico completo, de la habilidad disparo. Este apartado (clasificación del tipo de disparo) es uno de los aportes del presente ejercicio investigativo.

Superficie de contacto: La superficie de contacto empeine es la más reconocida en todos y cada uno de los trabajos investigativos en los últimos 10 años, porque antes de esto, Álvarez et al. (2018) demostraban que, en el borde interno, obtenía el valor más alto (42\%) en participación de los goles o F.É., frente al empeine (36\%).
Estos datos, se modificaron en los últimos años hasta el presente estudio, donde se analizan 24 equipos nacionales de la copa mundial 2016 y donde la superficie de contacto empeine $(51,42 \%)$, sigue marcado la direccionalidad. Datos confirmados por un sinnúmero de escritos (Álvarez et al. 2018; Candelas et al. 1997; Irokawa et al. 2010; García et al. 2006), de los cuales, se podrían destacar valores similares, como por ejemplo $(75,9 \%)$, en el presentado por Lapresa et al. (2013), en el estudio de cinco partidos de España en la Euro 2010 y con alguna diferencia, pero en la misma línea (75,9\%), encontrado por Álvarez (2015), en su tesis, el cual, analizó un solo equipo nacional.

Entre tanto, resultados encontrados en la misma dirección (Candelas et al. 1997), las diferencias se encuentran en la superficie del borde interno (CIN) (31,82\%), distante del 6,1\% (Álvarez, 2015) y del 5,9\%, de Lapresa et al. (2013). Álvarez planteaba que el $33,4 \%$ de técnicas utilizadas eran con borde interno (CIN), pero se refería a toda la cantidad de acciones de juego que suceden en el partido. Por lo tanto, la superficie de contacto empeine (EMP) es considerada como la superficie más utilizada de las F.É. en el Fútsal (García et al. 2006); se puede precisar que, desde el punto de vista de la acción ofensiva, específicamente en la F.É., las acciones de disparo utilizan el empeine (EMP), en un 51,42\%, donde se coincide con Lapresa et al. (2013), quien determinaba la utilización del empeine, con $75,9 \%$.

En cuanto a la superficie de contacto punta (CPU) $(9,01 \%)$ es poco utilizada en la F.É. Se han encontrado resultados con ciertas diferencias como, por ejemplo, lo expuesto por De Melo (2010), en su estudio de las finalizaciones en equipos juveniles, quien aseguraba que el fundamento técnico de la punta en el tiro o disparo alcanzaba hasta el 19,2\%; entre tanto que, Lapresa et al. (2013), analizando al equipo campeón de la UEFA 2010, determinaba la superficie de contacto punta (CPU), con un $15,6 \%$. Esto le permitía a Irokawa et al. (2010) en el estudio de las finalizaciones del mundial 2008, podía reconocerle a la superficie de contacto punta (CPU), como la superficie con la mayor precisión y mayor velocidad de salida para la F.É., pero que, para el caso del presente proceso investigativo, no se comprobó.

Dominancia: Cuando se ha estudiado la dominancia en las ejecuciones de las F.É., seguramente, se ha considerado el hecho que, la mayoría de los jugadores 
que practican este deporte, son diestros $(69,51 \%)$. Este valor lo obtuvo De Bortoli et al. (2002), cuando realizó el análisis a 6 equipos profesionales de Brasil. Se podría esperar la misma proporción al contabilizar las F.É. con cada pierna, es decir, que la mayoría de los goles o F.É., la deberían realizar los diestros.

Como ha ocurrido con otras variables, como la fase del torneo, en una relación de 1 a 1, por tanto, el resultado esperado en este estudio debería ser cercano al $70 \%$, pero no fue así; se encontró, que el $55,68 \%$ de las F.É., se realizaron con pierna derecha (el $80 \%$ de lo esperado), lo cual, indicaría, automáticamente, que los ambidiestros y los zurdos, estarían produciendo por encima de esa relación $1 \times 1$. Si se considera que el $30,5 \%$ de los jugadores son zurdos (De Bortoli et al. 2002), incluidos los ambidiestros o que manejan las dos piernas, se hace relevante el dato de $39,77 \%$ de las F.É., que se logran con pierna zurda, es decir, que una minoría del $30,49 \%$ de los jugadores que tienen esta condición desarrolla una efectividad del $130,45 \%$, significando que estos jugadores rinden para su equipo un porcentaje mayor que los diestros (los cuales, son la mayoría en el juego). Situación que es conjetura y que debiera ser estudiada más a fondo, para considerar que los jugadores zurdos y los ambidiestros presentan mayor rendimiento en efectividad frente a la F.É., que los jugadores diestros.

Perfil: Aun así, el presente estudio destaca un elemento técnico, que ha sido poco estudiado a fondo, hasta el momento (De Bortoli et al. 2002) y que permitirá explicar, una mejor utilización de los jugadores y de su pierna hábil dentro del campo o partido y es la consideración del perfil. El resultado encontrado, para este perfil, presenta que el $44,60 \%$ de las F.É., se realizan con perfil cambiado o contrario, perfil frontal $(33,81 \%)$ y solo el $21,59 \%$, es perfil propio, lo cual, pudiera explicar, que más importante que la dominancia (para este caso) es la utilización posicional en el campo. Al parecer, se pudiera estimar, como concluyente para el juego, que el perfil dominante en el fútsal no es el propio, sino por el contrario, es el perfil cambiado, es decir, que los jugadores buscan sectores centrales para la F.É. (De Bortoli et al. 2002).

\section{CONCLUSIÓN}

Valorando el desempeño del jugador ejecutor, a partir de la identificación de factor técnico, permite esclarecer las relaciones, asociaciones y patrones de las F.É., susceptibles de ser forjados en la preparación del deportista, donde debe conocer al oponente, coincidiendo con Campos et al. (2015), Drust (2010) y Serrano et al. (2013).

En la modalidad del Fútbol Sala, el factor técnico utilizado por el ejecutor en la F.É., está representado por cinco variables (habilidad técnica, tipo de disparo, superficie de contacto, dominancia y perfil), de las cuales, cada una destaca valores concluyentes. La habilidad técnica es el disparo, el tipo de disparo es el remate, variables que se siguen confirmando, a través de cada estudio investigativo.

Las superficies de contacto que se destacan en la F.É. son tres (empeine, planta y borde interno) y que coinciden, también, con las más utilizadas en el juego, según Álvarez (2015), a diferencia de las dos habilidades más utilizadas en el juego del fútbol (borde interno y empeine) (García et al. 2006).

La superficie de contacto más utilizada en las F.É. es el empeine, pero al discriminarlo por habilidades utilizadas, sorprende el hallazgo de la superficie de contacto del borde interno, cuando no hay presente la habilidad técnica previa, es decir, cuando el ejecutor define de primera intensión.

El perfil cambiado y la dominancia derecha son predominantes en la F.É. por el ejecutor; se pudiera explicar qué, más importante que la potencia y la dominancia, es la utilización posicional en el campo. Este fenómeno, pudiera tener explicación en la posibilidad que tiene el jugador de acomodar su propio perfil y dominancia a la hora de resolver la F.É.; esta consideración pudiera explicar, en parte, por qué se nota el aumento de la dominancia izquierda, para los ejecutantes de las F.É.

Los valores de variables categóricas del factor técnico son importantes, desde un análisis cuantitativo, pero se debe mencionar que la superficie de contacto cabeza y el tipo de disparo autogol, el doble penal, el penal y F.É., tienen valores tan bajos, que se desestiman en este tipo de análisis de las F.É., reiteramos, desde un análisis cuantitativo, lo cual, se hace necesario que este tipo de variables que, aparentemente, son desestimadas, por su no aparición se debe a otro tipo de explicación, por tanto, se tendrán que evaluar bajo análisis cualitativo, por el factor de importancia y de trascendencia en el juego. 
Conflictos de intereses: El manuscrito fue preparado y revisado con la participación de los autores, quienes declaramos que no existe ningún conflicto de intereses que ponga en riesgo la validez de los resultados presentados. Financiación: Este estudio fue financiado por la Institución Universitaria Escuela Nacional del Deporte I.U.E.N.D.

\section{REFERENCIAS}

1. ABDEL-HAKIM, H. 2014. Quantitative analysis of performance indicators of goals scored in the futsal world cup thailand 2012. Pamukkale Journal of Sport Sciences. 5(1):113-127.

2. AGRAS, H.; FERRAGUT, C.; ABRALDES, A. 2016. Match analysis in futsal: a systematic review. International Journal of Performance Analysis in Sport. 16(2):652-686. https://doi.org/10.1080/24748668.2016.11868915

3. ÁlVAREZ KUROGI, L. 2015. Análisis técnico-táctico de la fase ofensiva de la selección española de fútbol sala (Eurocopa, 2010). Tesis doctoral, Universidad de La Rioja (Logroño, España).

4. ÁlVAREZ MEDINA, J.; MURILlO LORENTE, V.; GARCÍA. F.; PARRA ARTAL, A. 2018. Análisis Observacional de los goles de dos temporadas de la LNFS. Revista Internacional de Medicina y Ciencias de la Actividad Física y el Deporte. 18(69):27-42.

https://doi.org/10.15366/rimcafd2018.69.002

5. ALVES, L. 2010. Descriptive study of the level of technical-tactical goalkeeper futsal world cup in 2008. Master's tesis, Federal University of Minas Gerais: School of Physical Education, Physiotherapy and Occupational Therapy, Brazil.

6. BLANCO, A.; LOSADA J.; ANGUERA M. 2001. Diseños observacionales, cuestión clave en el proceso de la Metodología Observacional. Revista Metodología de las Ciencias del Comportamiento. 3(2):135-160.

7. CAMPOS, F.; PELLEGRINOTTI, Í.; PASQUARELLI, N.; RABELO, N.; SANTA CRUZ, R.; GÓMEZ, M. 2015. Effects of game-location and quality of opposition in futsal league. International Journal of Performance Analysis in Sport. 15(2):598-607.

https://doi.org/10.1080/24748668.2015.11868817
8. CANDELAS, J.; LOZANO, J.; MORENO, M.; NIÑO, S.; RODRÍGUEZ, A. 1997. Técnica individual y colectiva Fútbol Sala. Curso Nivel 1 - Instructor de Futbol Sala. Imprenta Sarabia SL (España). p.97-115.

9. CUASAPUD, D.; HURTADO, H. 2018. Impacto del primer gol: Copa Mundial Futsal FIFA Colombia 2016. RBFF-Revista Brasileira de Futsal e Futebol (Brasil). 10(40):544-549.

10. DE BORTOL, A.; DE BORTOL, R.; MÁRQUE, S. 2001. Usar coeficientes ofensivos para analizar el rendimiento deportivo en fútbol sala. European Journal of Human Movement (España). (7):7-17.

11. DE BORTOLI, R.; DE BORTOLI, A.; MÁRQUEZ, S. 2002. Comparación entre posición declarada y eficacia subjetiva en fútbol sala. Red: Revista de Entrenamiento Deportivo (España). 16(1):23-26.

12. DE MELO, M. 2010. Perfil das finalizações no futsal: um estudo do xxii jogos da juventude do paraná. (Tesis de pregrado) Educación Física, Universidade Federal de Minas Gerais (Minas Gerais, Brasil).

13. DRUST, B. 2010. Performance analysis research: Meeting the challenge. Journal of Sports Sciences. 28(9):921-922.

https://doi.org/10.1080/02640411003740769

14. DUARTE, R. 2008. Análise da utilização da posse de bola durante o processo ofensivo no futsal. Contributo para a determinação da Eficiência Colectiva. Motricidade. 4(2):78-82.

15. FEDERACIÓN INTERNACIONAL DE FÚTBOL ASOCIADO, FIFA. 2016. Copa Mundial de Fútsal de la FIFA Colombia 2016. Disponible desde internet en: https://es.fifa.com/Fútsalworldcup/statistics /index.html (con acceso el 25/10/2016).

16. GARCÍA, A.; ZABALA, M.; LOZANO, L.; LOZANO, J.; SOTO, V. 2006. Análisis de los golpeos de empeine y puntera en jugadores de elite de fútbol-sala. Archivos de medicina del deporte (España). 23(114):274-282.

17. GARGANTA, J. 2009. Trends of tactical performance analysis in team sports: bridging the gap between research, training and competition. Rev Port Cien Desp. 9(1):81-89. https://doi.org/10.5628/rpcd.09.01.81 
18. GONÇALVES, B.; FIGUEIRA, B.; MACAS, V.; SAMPAIO, J. 2014. Effect of player position on movement behaviour, physical and physiological performances during an 11-a-side football game. Journal of Sports Sciences (España). 32(2):191-199.

https://doi.org/10.1080/02640414.2013.816761

19. HEADRICK, J.; DAVIDS, K.; RENSHAW, I.; ARAÚJO, D.; PASSOS, P.; FERNANDES, O. 2012. Proximityto-goal as a constraint on patterns of behaviour in attacker-defender dyads in team games. Journal of Sports Sciences (Gran Bretaña). 30(3):247-253.

https://doi.org/10.1080/02640414.2011.640706

20. IROKAWA, G.; SOARES, V.; ABURACHID, C.; GRECO, P.; SOUZA, C. 2010. Caracterización de las finalizaciones en el juego de Fútsal. Un estudio sobre la copa del mundo de Fútsal FIFA 2008. Trabajo de grado de pregrado, Profesional en Educación Física, Universidad Federal de Minas Gerais (Minas Gerais, Brasil).

21. LAPRESA, D.; ÁlVAREZ, L.; ARANA, J.; GARZÓN, B.; CABALLERO, V. 2013. Observational analysis of the offensive sequences that ended in a shot by the winning team of the 2010 UEFA Futsal Championship. Journal of Sports Sciences (España). 31(15):1731-1739.

https://doi.org/10.1080/02640414.2013.803584

22. LAPRESA, D.; CAMERINO, O.; CABEDO, J.; ANGUERA, M.; JONSSON, G.; ARANA, J. 2015. Degradación de T-patterns en estudios observacionales: Un estudio sobre la eficacia en el ataque de fútbol sala. Cuadernos de Psicología del Deporte (España). 15(1):71-82.

https://doi.org/10.4321/S1578

$-84232015000100007$

23. LEITE, W. 2013. Euro 2012: Analysis and Evaluation of Goals Scored. International Journal of Sports Science (España). 3(4):102-106.

https://doi.org/10.5923/j.sports.20130304.02

24. LIU, H.; GÓMEZ, M.; GONÇALVES, B.; SAMPAIO, J. 2016. Technical performance and match-tomatch variation in elite football teams. Journal of Sports Sciences (España). 34(6):509-518. https://doi.org/10.1080/02640414.2015. 1117121
25. MARCHI, R.; SILVA, C.; SCRAMIN, L.; TEIXEIRA, A.; CHIMINAZZO, J. 2010. Incidencia de goles resultantes de contraataques de equipos de fútbol sala. Revista de la Facultad de Educación Física de UNICAMP. 8(3):16-22.

26. MOORE, R.; BULLOUGH, S.; GOLDSMITH, S.; EDMONDSON, L. 2014. A Systematic Review of Futsal Literature. American Journal of Sports Science and Medicine (España). 2(3):108-116. https://doi.org/10.12691/ajssm-2-3-8

27. NEVILL, A.; ATKINSON, G.; HUGHES, M.; COOPER, S. 2002. Statistical methods for analysing discrete and categorical data recorded in performance analysis. Journal of Sports Sciences. 20(10):829-844. https://doi.org/10.1080/026404102320675666

28. PUENTE, J.; ÁlVAREZ, J.; MANERO, J.; MANONELLES, P. 2004. Análisis de las acciones ofensivas que resultan en el objetivo de la liga profesional del fútbol sala español. Revista de entrenamiento deportivo (España). 18(4):27-32.

29. SANTANA, W.; LAUDARI, B.; ISTCHUK, L.; ARRUDA, F. 2013. Análise dos Gols em Jogos de Futsal Feminino de Alto Rendimento. Revista Brasileira de Ciência e Movimento (Brasil). 21(4):157-165. http://dx.doi.org/10.18511/0103-1716/rbcm. v21n4p157-165

30. SARMENTO, H.; BRADLEY, P.; ANGUERA, M.; POLIDO, T.; RESENDE, R.; CAMPANICO, J. 2016. Quantifying the offensive sequences that result in goals in elite futsal matches. Journal of Sports Sciences. 34(7):621-629.

https://doi.org/10.1080/02640414.2015.1066024

31. SERRANO, J.; SHAHIDIAN, S.; SAMPAIO, J.; LEITE, N. 2013. The Importance of Sports Performance Factors and Training Contents From the Perspective of Futsal Coaches. Journal of Human Kinetics. 38:151-160. https://doi.org/10.2478/hukin-2013-0055 